\title{
A note on the zeros of the Bessel function
}

\author{
By R. Wilson.
}

(Received 2nd August, 1938. Read 4th November, 1938.)

This note contains an elementary proof of the well-known result that every solution of Bessel's equation

$$
x^{2} y^{\prime \prime}+x y^{\prime}+\left(x^{2}-n^{2}\right) y=0,
$$

with a real parameter $n$ has an infinite number of real positive zeros.

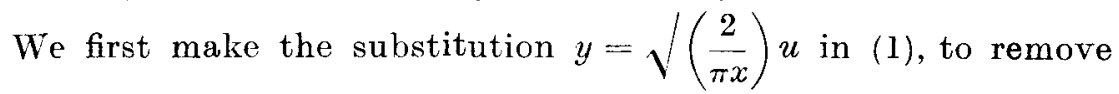
the first derivative, giving

$$
u^{\prime \prime}+\left[1+\left(\frac{1}{4}-n^{2}\right) / x^{2}\right] u=0 .
$$

This equation approximates, for $x$ sufficiently large, to the form $u^{\prime \prime}+u=0$ associated with the circular functions. In fact, when $n^{2}=\frac{1}{4}$, the solutions of (1) may be written

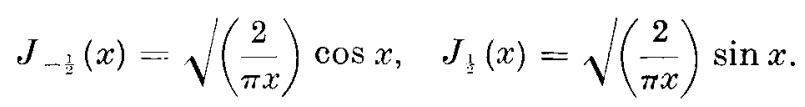

We employ the simple methods used by Bôcher ${ }^{1}$ in his studies of the Sturm-Liouville equation to exploit these facts.

Suppose first that $n^{2}-\frac{1}{4}<0$, and consider the equation

$$
v^{\prime \prime}+v=0 \text {. }
$$

The solutions of this equation are of the form $A \cos (x+a)$ and the zeros are at intervals of $\pi$. Let $x_{1}, x_{2}$ be two consecutive positive zeros of $v(x)$. Multiplying (2) by $v$ and (3) by $u$, and subtracting, we get

$$
\frac{d}{d x}\left(u^{\prime} v-u v^{\prime}\right)=u^{\prime \prime} v-u v^{\prime \prime}=\left(n^{2}-\frac{1}{4}\right) \frac{u v}{x^{2}}
$$

or

$$
\left[u^{\prime} v-u v^{\prime}\right]_{x_{1}}^{x_{u}}=-\left(\frac{1}{4}-n^{2}\right) \int_{x_{1}}^{x_{*}} u v \frac{d x}{x^{2}} .
$$

Two cases are possible: either $v(x)$ is positive between $x_{1}$ and $x_{2}$ with $v^{\prime}\left(x_{1}\right)>0$ and $v^{\prime}\left(x_{2}\right)<0$ or $v(x)$ is negative between $x_{1}$ and $x_{2}$ with $v^{\prime}\left(x_{1}\right)<0$ and $v^{\prime}\left(x_{2}\right)>0$.

1 M. Bôcher, Leçons sur les Méthodes de Sturm (Paris, 1917), Ch. III. and IV. 
Let us assume that $u(x)$ does not vanish between $x_{1}$ and $x_{2}$. Then, from (4), in the first case the left-hand side has the same sign as $u(x)$ while the right-hand side has the opposite sign to that of $u(x)$. This contradiction shows that $u(x)$ must vanish at least once between $x_{1}$ and $x_{2}$. In the second case the left-hand side has the sign of $-u(x)$ while the right-hand side has the sign of $u(x)$, again leading to a contradiction.

Next suppose that $n^{2}-\frac{1}{4}>0$, and consider the equation

$$
v^{\prime \prime}+\left[1-\left(n^{2}-\frac{1}{4}\right) / k^{2}\right] v=0,
$$

in which $k^{2}>\left(n^{2}-\frac{1}{4}\right)$. The solutions of this equation have their zeros separated by intervals of $\pi k / \sqrt{ }\left(k^{2}-n^{2}+\frac{1}{4}\right)$. Applying to (2) and (5) the same treatment as before, we get

$$
\left[u^{\prime} v-u v^{\prime}\right]_{x_{1}}^{x_{2}}=-\left(n^{2}-\frac{1}{4}\right) \int_{x_{1}}^{x_{2}} u v\left(\begin{array}{c}
1 \\
k^{2}
\end{array}-\frac{1}{x^{2}}\right) d x
$$

and, provided that $x_{2}>x_{1}>k>0$, the same argument holds as before. Thus, the real positive zeros of the solutions of (1) and (2) are at least as numerous as those of a cosine function.

Reverting to the case in which $n^{2}-\frac{1}{4}<0$ and making assumptions on $u(x)$ similar to those made on $v(x)$ (following (4)), we can now use $(2),(5)$ and $(6)$ to show that, for $x$ sufficiently large, $v(x)$ vanishes at least once between every pair of consecutive zeros of $u(x)$.

Similarly, we can use (2), (3) and (4) to obtain the same result when $n^{2}-\frac{1}{4}>0$.

The zeros of (5) occur with less frequency than those of (2), when $n^{2}-\frac{1}{4}>0$ and more frequently when $n^{2}-\frac{1}{4}<0$, but, by taking $k$ sufficiently large we can make the period of the solutions of (5) as near to $2 \pi$ as we like. From this and the remarks following (6) we see that the real and positive zeros of any solution of (1) approximate to those of $\cos (x+a)$, for $x$ sufficiently large, a matter indicated by the nature of the asymptotic expansion ${ }^{1}$ of $J_{n}(x)$.

1 T. M. MacRobert, Spherical Harmonics (London, 1927), 274.

\section{University College,} Swansea. 